\title{
Rede de apoio, eventos estressores e mau ajustamento na vida de crianças e adolescentes em situação de vulnerabilidade social*
}

\author{
Social networks, stressful events and maladjustment in \\ the lives of vulnerable children and adolescents
}

Recibido: febrero 2 de 2011 | Revisado: julio 22 de 2011 | Aceptado: noviembre 24 de 2011

\author{
Normanda Araujo DE Morais ** \\ Universidade de Fortaleza, Brasil \\ SÍlviA HELENA KOLLER **** \\ Universidade Federal do Rio Grande do Sul, Brasil \\ MARCELA RAFFAELLI ***** \\ University of Illinois at Urbana-Champaign, United Stated
}

SICI: 1657-9267(201209)11:3<779:RDAEEA>2.0.TX;2-Z

Para citar este artículo. Morais, N. A., Koller, S. H. \& Raffaelli, M. (2012). Rede de apoio, eventos estressores e mau ajustamento na vida de crianças e adolescentes em situação de vulnerabilidade social. Universitas Psychologica, 11 (3), 779-791.

* Universidade de Fortaleza (UNIFOR), Brasil. Esse artigo é parte da dissertação de Doutorado da primeira autora, sobre orientação da segunda autora e co-orientação da terceira autora. As autoras agradecem à CAPES (Coordenação de Aperfeiçoamento de Pessoal de Nível Superior) pelas bolsas de doutorado e de doutorado sanduíche concedidas à primeira autora para a realização do seu doutoramento.

** Endereço: Universidade de Fortaleza (UNIFOR), Avenida Washington Soares, 1321, PPG-Psicologia (Bloco N, Sala 13), Fortaleza, CE CEP: 60811-905. Email: normandaaraujo@gmail.com

**** silvia.koller@gmail.com ResearcherID: Koller, S. B-2629-2008

****** mraffael@illinois.edu
RES UMO

O estudo comparou dois grupos de crianças e adolescentes $(N=98 ; 11-18$ anos) em situação de vulnerabilidade social (G1 em situação de rua e G2 vivia com família) quanto às variáveis: rede de apoio, eventos estressores e mau ajustamento. Avaliou, ainda, o efeito de moderação das características (tamanho e proximidade) da rede de apoio dos participantes sobre a associação entre eventos estressores e mau ajustamento. Os dados foram coletados com o método de inserção ecológica em instituições e com o uso de instrumentos padronizados. Houve diferença de composição nas redes de apoio dos grupos: G1 possui mais contatos e maior fator de proximidade no campo de amigos/vizinhos/parentes que G2; maior média de eventos estressores e o pior indicador de ajustamento. Proximidade familiar moderou o ajustamento de quem vivencia alto nível de eventos estressores. Programas sociais que visem ao atendimento de famílias em situação de vulnerabilidade social devem ser priorizados.

\section{Palavras-chave}

Rede de apoio; fator de proteção; vulnerabilidade social; família.

Palavras-chave plus

Estudo transversal, psicologia social, pesquisa quantitativa.

\section{A B S T R A C T}

Two groups of at risk children and adolescents $(N=98 ; 11-18$ years $)$ were compared in terms of social networks, stressful events, and maladjustment and the moderating effects of social network characteristics (size and proximity) on the association between stressful life events and maladjustment evaluated. G1 consists of street adolescents and G2 of those living with their families. Data were collected using the ecological engagement method and standardized instruments. Results indicated group differences in characteristics of social networks: street adolescents (G1) had more contacts and proximity with friends/neighbors/relatives than youth in family situations (G2). Street adolescents also reported higher levels of stressful events and maladjustment. Family proximity moderated the association between stressful life events and maladjustment, suggesting that social programs should be provided to strengthen vulnerable families.

Key words authors

Social Network, Protective Factors, Social Vulnerability, Family.

Key words plus

Cross-Sectional Study, Social Psychology, Quantitative Research. 
Na literatura da Psicologia do Desenvolvimento, os termos fator de risco e fator de proteção têm sido cada vez mais utilizados para descrever os processos desenvolvimentais durante o ciclo vital, especialmente de pessoas que vivem em situação de vulnerabilidade social.

Fatores de risco são aquelas condições ou variáveis que estão associadas com a maior probabilidade de resultados desenvolvimentais negativos ou indesejáveis (Cowan, Cowan, \& Schulz, 1996; Jessor et al., 1995). Uma revisão dos modelos de risco, elaborada por Martins (2004), mostrou que a trajetória da evolução desse conceito evoluiu do chamado "modelo simples de risco" para o "modelo complexo de risco". No primeiro modelo, incluem-se os estudos considerados limitados na conceitualização do risco, pois defendem uma visão estática, causal e linear entre risco e mau ajustamento. $\bigcirc$ modelo complexo de risco surgiu na década de 80 , com as pesquisas sobre fatores de proteção e resiliência. Propunham o risco como processo, não considerado per se, mas em relação a variáveis protetoras, que seriam antagonistas à emergência dos problemas precipitados pelos fatores de risco.

Variáveis protetivas podem estar presente e moderar, amortecer (buffer), mediar ou isolar o risco, tal como descreveu Jessor et al. (1995). Os fatores de proteção explicariam, portanto, como diferentes indivíduos, submetidos às mesmas condições adversas, alcançariam resultados desenvolvimentais diversos, uma vez que são eles os responsáveis por fazer a mediação entre a experiência do risco e tais resultados. A mediação, de acordo com Fergus e Zimmerman (2005) poderia agir em dois sentidos:

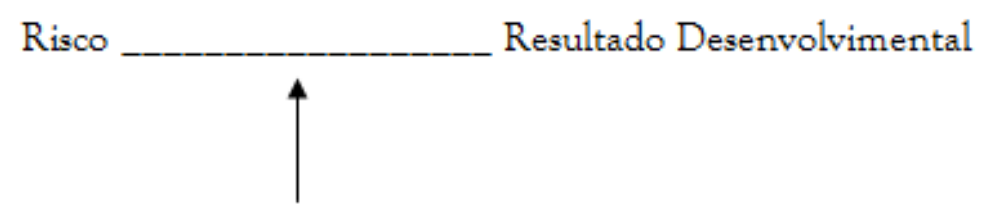

Fatores de Proteção

\section{Modelo Protetivo-Estabilizador}

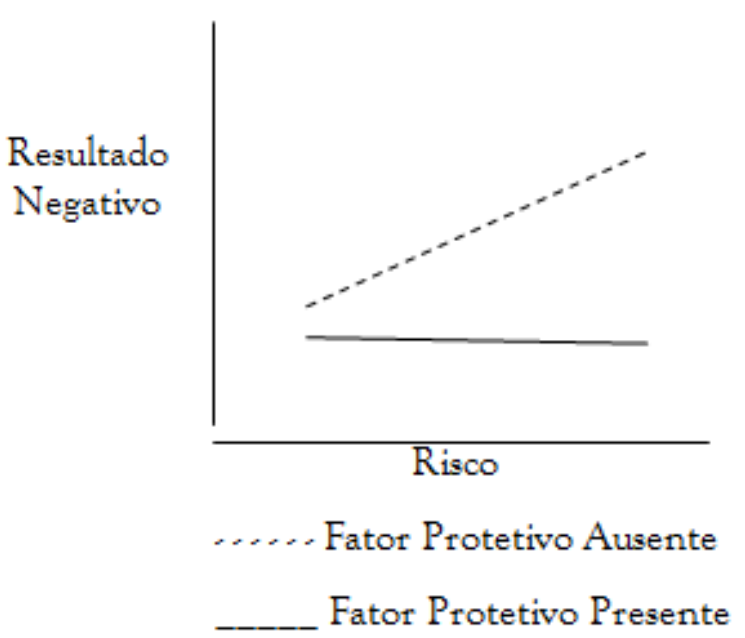

Modelo Protetivo-Reativo

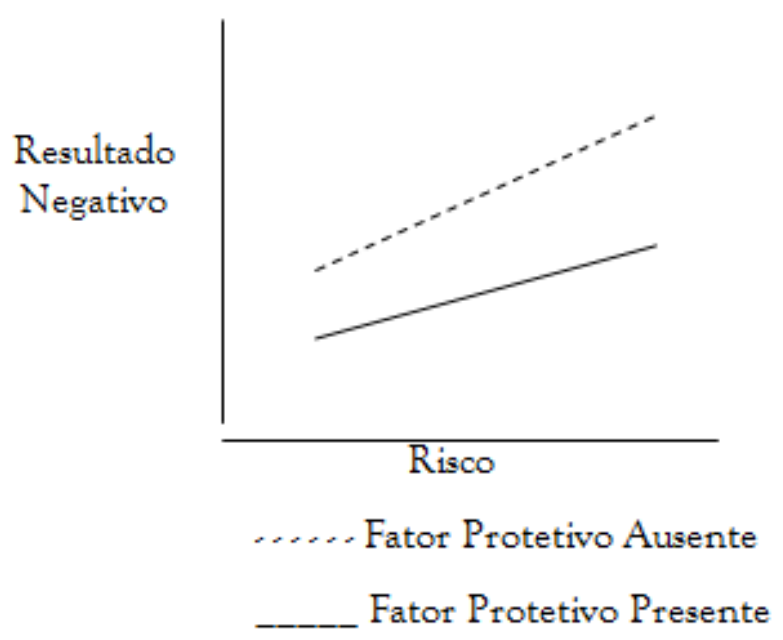

Figura 1. Modelo de influência dos fatores de proteção (baseado em Fergus \& Zimmerman, 2005, p. 402) 
produzindo um resultado positivo ou reduzindo/ evitando um resultado negativo.

Alguns (ou todos) fatores de proteção (Masten \& Garmezy, 1985) podem estar presentes diante do risco: atributos disposicionais das pessoas (auto -estima, auto-eficácia, por exemplo); laços afetivos no sistema familiar e/ou em outros contextos que ofereçam suporte emocional em momentos de estresse; e, sistemas de suporte social, seja na escola, no trabalho, nos centros religiosos, no serviço de saúde, que propiciem competência e determinação individual e um sistema de crenças para a vida.

Fergus e Zimmerman (2005) propuseram a classificação dos fatores de proteção em dois grupos: patrimônio (assets) e recursos (resources). Patrimônio inclui fatores internos ao indivíduo, tais como competência, habilidades sociais e auto-eficácia. Os recursos seriam externos, como apoio parental, comunitária ou institucional e monitoramento de uma figura adulta. Características individuais ( $p a-$ trimônio) ou da rede de apoio (recursos) moderam ou reduzem os efeitos do risco sobre um resultado desenvolvimental.

A Figura 1 ilustra como os fatores de proteção operam para alterar a trajetória da exposição ao risco, levando a resultados positivos (modelo protetivo-estabilizador) ou menos negativos (modelo protetivo-reativo; Fergus \& Zimmerman, 2005; Luthar, Cicchetti \& Becker, 2000). Pelo modelo protetivo-estabilizador, o fator protetivo neutraliza os efeitos do risco. Altos níveis de risco são associados com resultados desenvolvimentais negativos quando o fator protetivo está ausente e, positivos, quando presente. No modelo protetivo-reativo, a relação entre o risco e o resultado adverso é mais forte quando o fator protetivo está ausente. $\mathrm{Na}$ presença deste, ao contrário, embora o resultado adverso ainda se mostre presente, terá menor intensidade. Um modelo protetivo-estabilizador existe, por exemplo, para adolescentes que, mesmo tendo amigos usuários de droga, possuem apoio familiar que os protegem de iniciar também o uso (Farrel \& White, 1998). Um modelo protetivo-reativo apareceria nos casos em que a família detectaria o uso ainda no início e agiria no sentido de minimizar consequências.

\section{Rede de Apoio Social como Fator de Proteção}

De acordo com Brito e Koller (1999), tal rede consiste num conjunto de sistemas e de pessoas significativas que compõem os elos de relacionamento recebidos e percebidos do indivíduo. Bates e Toro (1999) especificaram a definição de apoio social como sendo interações pelas quais o indivíduo ou grupo fornece diretamente ao(s) outro(s) conexão, recursos (informação, conselho e assistência instrumental) e validação/afirmação (noção de que o indivíduo é escutado e cuidado e que tem suas percepções, sentimentos, pensamentos e comportamentos valorizados).

A rede de apoio deve ser vista como um processo contínuo, na medida em que a pessoa se insere em novas e diferentes ecologias. $\mathrm{O}$ indivíduo tanto recebe influências da rede de apoio quanto influencia a mesma, participando da sua construção (Bronfenbrenner, 2005). Por exemplo, fatores individuais e menor disponibilidade de apoio (na família, em grupos) podem contribuir para que a criança/adolescente não desenvolva habilidades sociais (Samuelsson, Thernlund \& Ringström, 1996; Siqueira, Tubino, Schwarz \& Dell’Aglio, 2009).

Dois modelos teóricos ("amortecedor" do evento estressor - buffering model e efeito direto ou principal - direct/main-effect model) explicam os mecanismos pelos quais o apoio social age como proteção (Bates \& Toro, 1999; Cohen \& Wills, 1985; Samuelsson, Thernlund \& Ringström, 1996). No modelo "amortecedor", o apoio social promove efeitos benéficos de adaptação/ajustamento apenas em circunstâncias nas quais os indivíduos são expostos a altos níveis de estresse e/ou eventos frequentes ou de alto impacto. No modelo do efeito direto ou principal, o apoio social age sem levar em conta os níveis de estresse aos quais os indivíduos estão expostos. Portanto, para este modelo o apoio social é benéfico para todos, sendo associada à saúde e ao bem-estar dos indivíduos, sendo um fator fundamental para a adaptação ao estresse e suscetibilidade a distúrbios físicos e emocionais (Masten \& Garmezy, 1985; Rutter, 1987; Samuelsson, Thernlund \& Ringström, 1996). 
Em uma revisão sobre a relação entre rede de apoio e problemas comportamentais em crianças/adolescentes em situação de rua, Johnson, Whitbeck e Hoyt (2005) concluíram que uso de substância, depressão, delinquência e abuso físico e sexual têm sido relacionados com características da rede de apoio social. Crianças/adolescentes de rua que relatam redes pequenas e transitórias são mais expostos a (ou engajam-se mais em) comportamentos de alto-risco. Tal rede de apoio social de crianças em situação de rua (quando adequada) pode amortecer o estresse e os perigos da vida na rua e, alternativamente (quando inadequada), expô-los a comportamentos de risco e vitimização. No entanto, não fica claro o que definem como uma rede adequada ou não. Como os adolescentes geralmente fogem de casa de famílias abusivas e desorganizadas, a literatura descreve suas redes de apoio social como homogêneas em natureza, embora transitórias, orientadas para a rua, compostas por pares e com pouco contato familiar. Características da rede de apoio social (como disponibilidade para proteção) de pessoas pobres também têm sido associadas com mau ajustamento (uso de drogas, comportamento violento e comportamento sexual; ver revisão Fergus \& Zimmerman, 2005). Conectividade entre membros da família, monitoramento e comunicação aberta parental, renda, envolvimento, realização e orientação positiva com escola, participação em atividades extracurriculares, comunitárias e religiosidade podem ser listados como fatores de proteção, mas para cada um deles, formas específicas e influência diferenciados devem ser levados em conta para análise de mau ajustamento.

Portanto este artigo tem como objetivo: 1) descrever e comparar dois grupos de adolescentes em situação de vulnerabilidade social (um grupo em situação de rua e um grupo que vive com sua família) quanto à sua rede de apoio social e afetiva, ao número e impacto dos eventos estressores e ao indicador geral de mau ajustamento; e 2) verificar se as características da rede de apoio (tamanho e qualidade) moderaram o efeito do número e impacto dos eventos estressores sobre o índice geral de mau ajustamento.

\section{Método}

\section{Delineamento e Participantes}

Trata-se de um estudo transversal que foi realizado com 98 crianças/adolescentes, em dois grupos: aqueles que vivem em situação de rua $(N=32 ; 32.7 \%)$ e os que moram com suas famílias e frequentam uma instituição para jovens em situação de vulnerabilidade social ( $N=66 ; 67.3 \%)$. No grupo de base-rua (G1), a maioria ( $N=27 ; 84.4 \%$ ) era do sexo masculino, com idade média de 14.69 anos ( $\mathrm{SD}=1.85)$. Já no grupo com base-familiar (G2), 38 (57.6\%) eram do sexo feminino e tinham em média 15.53 anos $(S D=1.12)$.

\section{Instrumentos}

Foram utilizados três blocos de instrumentos (descritos na Tabela 1), os quais avaliaram fator de risco, fator de proteção e ajustamento.

Para realização das análises, foi criada, ainda, outra variável: o Indicador Geral de Mau Ajustamento. Trata-se de um compósito formado a partir da soma dos seis indicadores de ajustamento (sintomas físicos; uso de drogas; comportamento suicida; comportamento sexual de risco; afeto positivo e afeto negativo). Para sua criação, foram realizados os seguintes passos: 1) a inversão dos itens da Escala de Afeto Positivo, de forma que os escores mais altos representassem menor afeto positivo, ou seja, maior "mau ajustamento"; 2) a transformação de cada indicador de ajustamento em um escore padronizado (escore $z$ ), o qual permitiu a comparação dessas medidas, obtidas a partir de diferentes escalas de mensuração; e 3) a soma dos escores padronizados (escore $z$ ) de todos os indicadores a fim de formar o indicador geral de mau ajustamento. Em se tratando de um indicador que é negativo (mau ajustamento), quanto mais positivo o valor da medida, pior o ajustamento (maior o escore em mau ajustamento). Quanto mais negativo, porém, melhor o ajustamento (pior o escore em mau ajustamento).

\section{Procedimentos}

Os participantes foram recrutados em instituições de atendimento para adolescentes em situação de 
TABELA 1

Definição e descrição dos Instrumentos Utilizados

\begin{tabular}{|c|c|c|c|}
\hline Dimensão/variável & Instrumento & Descrição & Valores \\
\hline \multicolumn{4}{|l|}{ A. Fator de Risco } \\
\hline Eventos Estressores & $\begin{array}{l}\text { Inventário de Eventos } \\
\text { Estressores (adaptado de } \\
\text { Kristensen, Dell'Aglio, Leon, } \\
\text { \& D'Incao, 2004). }\end{array}$ & $\begin{array}{l}61 \text { itens que avaliam a presença/ } \\
\text { ausência de eventos de vida } \\
\text { estressores, assim como o impacto. }\end{array}$ & $\begin{array}{l}\text { - Número - }(0-61) \\
\text { - Impacto - }(1-5)\end{array}$ \\
\hline \multicolumn{4}{|l|}{ B. Fator de Proteção } \\
\hline Rede de Apoio Social & $\begin{array}{l}\text { Mapa dos Cinco Campos } \\
\text { (Hoppe, 1998; Samuelsson, } \\
\text { Thernlund \& Ringström,1996) }\end{array}$ & $\begin{array}{l}\text { Avalia número de contatos e fator } \\
\text { de proximidade da rede de apoio } \\
\text { em cinco contextos (família, escola, } \\
\text { instituição, parentes/amigos/vizinhos } \\
\text { e contatos formais). }\end{array}$ & $\begin{array}{l}\text { - Número de contatos } \\
-\left(0-109^{4}\right) \\
\text { - Fator de } \\
\text { Proximidade }^{5}-(0-8)\end{array}$ \\
\hline \multicolumn{4}{|c|}{ C. Indicadores de Ajustamento } \\
\hline Sintomas Físicos & $\begin{array}{l}\text { Checklist de Sintomas Físicos } \\
\text { (Morais, Koller \& Raffaelli, } \\
\text { 2010) }\end{array}$ & $\begin{array}{l}\text { Os participantes relataram a } \\
\text { presença de } 11 \text { sintomas (febre, dor } \\
\text { de cabeça, problema respiratório } \\
\text { etc.) durante o último mês }\end{array}$ & $\begin{array}{l}0 \text { - nenhum sintoma } \\
\text { a } \\
11 \text { - todos os } \\
\text { sintomas. }\end{array}$ \\
\hline Uso de Drogas & $\begin{array}{l}\text { Escala sobre o Uso de Drogas } \\
\text { (baseada em Noto et al, 2004). } \\
(\text { Alpha }=0.87) .\end{array}$ & $\begin{array}{l}\text { Avalia a média do uso geral de } \\
\text { drogas (lícitas e ilícitas) no último } \\
\text { mês pelos participantes. }\end{array}$ & $\begin{array}{l}1 \text { - não usou a } 4- \\
\text { usou quase todos os } \\
\text { dias ( } 20 \text { dias ou mais). }\end{array}$ \\
\hline $\begin{array}{l}\text { Comportamento } \\
\text { Sexual de Risco }\end{array}$ & $\begin{array}{l}\text { Índice geral de } \\
\text { comportamento sexual de risco } \\
\text { (Morais, Koller \& Raffaelli, } \\
\text { 2010) }\end{array}$ & $\begin{array}{l}\text { Os participantes relataram } \\
\text { a presença dos seguintes } \\
\text { comportamentos: já ter transado na } \\
\text { vida, ter tido três parceiros ou mais } \\
\text { no último ano, ter transado antes } \\
\text { dos } 13 \text { anos, não ter usado camisinha } \\
\text { na última relação sexual e ter tido } \\
\text { alguma DST no último ano }\end{array}$ & $\begin{array}{l}0 \text { - nenhum risco a } \\
5 \text { - presença de todos } \\
\text { os comportamentos } \\
\text { sexuais de risco. }\end{array}$ \\
\hline $\begin{array}{l}\text { Comportamento } \\
\text { Suicida }\end{array}$ & $\begin{array}{l}\text { Escore de Risco para o } \\
\text { Comportamento Suicida } \\
\text { (Morais, Koller \& Raffaelli, } \\
\text { 2010). }\end{array}$ & $\begin{array}{l}\text { O escore foi criado através da junção } \\
\text { das questões de se "já pensou, já } \\
\text { tentou e quantas vezes tentou o } \\
\text { suicídio". }\end{array}$ & $\begin{array}{l}0 \text { - Não pensou e não } \\
\text { tentou; } 1 \text { - Pensou } \\
\text { e não tentou; } 2 \text { - } \\
\text { Tentou uma vez e, } \\
3 \text { - Tentou duas vezes } \\
\text { ou mais. }\end{array}$ \\
\hline Afeto Positivo & $\begin{array}{l}\text { Versão adaptada do PANAS-C } \\
\text { (Giacomoni, 2002; Laurent et } \\
\text { al., 1999). (Alpha = 0.88) }\end{array}$ & $\begin{array}{l}20 \text { itens; Os participantes indicaram, } \\
\text { o quanto se sentiam felizes, alegres, } \\
\text { animados etc. }\end{array}$ & $\begin{array}{l}1 \text { - nenhum pouco a } \\
5 \text { - muitíssimo }\end{array}$ \\
\hline Afeto Negativo & $\begin{array}{l}\text { Versão adaptada do PANAS-C } \\
\text { (Giacomoni, 2002; Laurent et } \\
\text { al., 1999). (Alpha = 0.92) }\end{array}$ & $\begin{array}{l}20 \text { itens; Os participantes indicaram, } \\
\text { o quanto se sentiam tristes, } \\
\text { deprimidos, impaciente etc. }\end{array}$ & $\begin{array}{l}1 \text { - nenhum pouco a } \\
5 \text { - muitíssimo }\end{array}$ \\
\hline
\end{tabular}

4 Número máximo de contatos apontado por um adolescente nesse estudo. Portanto, variável entre pesquisas.

5 Informações sobre o cálculo do fator de proximidade podem ser encontradas no artigo de Siqueira et al. (2009).

Fuente: elaboración propia.

vulnerabilidade social. Os dados do grupo com base-familiar foram coletados em uma ONG da periferia de Porto Alegre, caracterizado pela pobreza, violência e migração de crianças/adolescentes para o centro da cidade. Os dados do grupo de base-rua foram coletados em duas instituições da rede de 
TABELA 2

Comparação entre os Grupos de Base-Rua e Base-Familiar para as Variáveis da Rede de Apoio Social e Afetiva Total e por Contexto de Desenvolvimento (Família, Instituição, Escola, e Amigos/Vizinhos/Parentes)

\begin{tabular}{|c|c|c|c|c|}
\hline \multirow{2}{*}{ Variáveis } & & \multicolumn{2}{|c|}{$\mathrm{M}(\mathrm{SD})$} & \multirow{2}{*}{$t / U$} \\
\hline & & Base-Rua $^{\text {a }}$ & Base-Familiar $^{\mathrm{b}}$ & \\
\hline \multirow[t]{2}{*}{ Total } & Número total de contatos ${ }^{\mathrm{d}}$ & $42.15(20.22)$ & $40.61(17.51)$ & \\
\hline & Fator de proximidade ${ }^{c}$ & $6.25(1.37)^{*}$ & $5.40(1.21)$ & $t=-2.50 ; d f=66$ \\
\hline \multirow[t]{2}{*}{ Família } & Número total de contatos ${ }^{\mathrm{d}}$ & $8.84(4.31)$ & $8.69(4.34)$ & \\
\hline & Fator de proximidade ${ }^{c}$ & $6.59(1.44)$ & $5.89(1.45)$ & \\
\hline \multirow[t]{2}{*}{ Instituição } & Número total de contatos ${ }^{\mathrm{d}}$ & $13.47(6.94)^{* *}$ & $8.30(4.13)$ & $U=243 ; z=-3.04$ \\
\hline & Fator de proximidade ${ }^{\mathrm{d}}$ & $6.30(1.71)$ & $5.64(1.65)$ & \\
\hline \multirow[t]{2}{*}{ Escola } & Número total de contatos ${ }^{\mathrm{d}}$ & $6.31(5.04)$ & $8.61(4.82)$ & \\
\hline & Fator de proximidade ${ }^{c}$ & $5.93(1.89)$ & $5.08(1.59)$ & \\
\hline \multirow[t]{2}{*}{ Amigos,vizinhos e parentes } & Número total de contatos ${ }^{\mathrm{d}}$ & $10.26(10.67)$ & $10.38(6.34)$ & \\
\hline & Fator de proximidade ${ }^{c}$ & $6.81(1.53) * * *$ & $5.06(1.43)$ & $t=-4.26 ; d f=64$ \\
\hline
\end{tabular}

Nota. ${ }^{\mathrm{a}} \mathrm{N}=19$ e ${ }^{\mathrm{b}} \mathrm{N}=49$; ' Estatística paramétrica (Teste $t$ de Student); ${ }^{\mathrm{d}}$ Estatística não-paramétrica (Teste de Mann-Whitney); $* \mathrm{p}<0.05 ; * \mathrm{*}<<0.005 ; * * *_{p}<0.001$

Fuente: elaboración propia

atendimento especializada de Porto Alegre (abrigo diurno e albergue noturno).

O processo de coleta de dados teve como base o procedimento da Inserção Ecológica (Cecconello \& Koller, 2003; Eschiletti-Prati et al., 2008), a qual consiste em uma operacionalização metodológica da Abordagem Bioecológica do Desenvolvimento Humano (Bronfenbrenner, 1979/1996-1989; Bonfenbrenner \& Morris, 1998). Nesta pesquisa, a inserção ecológica consistiu em duas visitas por semana a cada instituição por sete meses. A primeira autora engajou-se em atividades do cotidiano de cada espaço, procurando conhecer e fazer parte das mesmas, ao mesmo tempo em que se vinculava com adolescentes e educadores das instituições. Tal vinculação garante validade ecológica aos dados, possibilitada pela relação de reciprocidade e complexização das atividades conjuntas em uma base regular de tempo. Com a maior vinculação entre a pesquisadora e os participantes, o procedimento de aplicação dos instrumentos pôde ter início.

No grupo de adolescentes em situação de rua, todos os instrumentos foram aplicados individual- mente, sendo que cada um levou três encontros (duração média de uma hora). No grupo com base-familiar foram necessários dois encontros com cada adolescente, sendo que o primeiro (aplicação coletiva, em grupos de 15 adolescentes, dos instrumentos em geral) durou em média 45 minutos e o segundo (entrevista individual) durou em média uma hora

\section{Aspectos éticos}

Esse projeto recebeu aprovação do Comitê de Ética da Universidade Federal do Rio Grande do Sul (Protocolo n. 2006533). Além disso, cada dirigente das instituições participantes assinou o Termo de Concordância para a realização da pesquisa. Por fim, cada participante recebeu informações sobre os objetivos da pesquisa, informações sobre o sigilo de sua identidade, confidencialidade dos dados, sobre o direito de aceitar participar ou não do estudo e/ou de desistir a qualquer momento. Após essa explicação, cada participante assinou o Termo de Consentimento Livre e Esclarecido. 
TABELA 3

Comparação de Médias entre os Grupos de Base-Rua e Base-Familiar para as Variáveis de Eventos Estressores (Número e Impacto), Indicadores de Ajustamento e o Indicador Geral de Mau Ajustamento

\begin{tabular}{|c|c|c|c|c|}
\hline \multirow{2}{*}{ Variáveis } & & \multicolumn{2}{|c|}{$M(S D)$} & \multirow{2}{*}{$\mathrm{t} / \mathrm{U}$} \\
\hline & & Base-Rua $^{a}$ & Base-Familiar ${ }^{\mathrm{b}}$ & \\
\hline \multirow[t]{2}{*}{ Eventos estressores } & Númeroc $(1-61)$ & $28.22(14.35)^{* * *}$ & $18.76(9.35)$ & $(t=-3.39 ; d f=44)$ \\
\hline & $\operatorname{Impacto}^{\mathrm{d}}(1-5)$ & $3.55(0.72)^{*}$ & $3.09(0.97)$ & $(U=605 ; z=-2.28)$ \\
\hline \multirow[t]{6}{*}{ Indicadores de ajustamento } & Sintomas físicos ${ }^{\mathrm{d}}(0-11)$ & $4.73(2.97)^{* *}$ & $2.58(1.64)$ & \\
\hline & Uso de drogas ${ }^{\mathrm{d}}(1-4)$ & $1.88(0.74)^{* * *}$ & $1.14(0.3)$ & \\
\hline & C. suicida $^{\mathrm{d}}(0-3)$ & $1(1.23)^{*}$ & $0.35(0.56)$ & \\
\hline & $\begin{array}{l}\text { C. sexual de risco } \\
(0-5)\end{array}$ & $1.52(1.12)^{* *}$ & $0.75(0.86)$ & \\
\hline & Afeto positivo ${ }^{c}(1-5)$ & $3.99(0.62) *$ & $3.75(0.5)$ & \\
\hline & Afeto negativo ${ }^{c}(1-5)$ & $2.88(0.9)^{* * *}$ & $2.13(0.66)$ & \\
\hline \multicolumn{2}{|c|}{ Indicador geral de mau ajustamento ${ }^{\mathrm{d}}$} & $2.92(4.99) * * *$ & $-1.35(2.37)$ & $(U=218 ; z=-3.45)$ \\
\hline
\end{tabular}

Nota. ${ }^{\text {a }} \mathrm{N}=21-30$, devido a dados faltando. ${ }^{\mathrm{b}} \mathrm{N}=48$ a 64 , devido a dados faltando; ${ }^{\mathrm{c}}$ Estatística paramétrica - Teste $\mathrm{t}$ de Student; ${ }^{\mathrm{d}}$ Estatística não-paramétrica - Teste de Mann-Whitney; ${ }^{*} p<0.05 ; * * p<0.005 ; * * * p<0.001$

Fuente: elaboración propia

\section{Resultados}

Análise Comparativa entre os Grupos - Rede de Apoio, Eventos Estressores e Mau Ajustamento

A Tabela 2 apresenta uma comparação da rede de apoio de G1 e G2. A comparação das médias entre os grupos mostrou diferença estatisticamente significativa na variável rede de apoio. $\mathrm{O}$ grupo de base-rua (G1) apresentou maior número de contatos no campo "instituição"; maior fator de proximidade total e no campo "amigos/vizinhos e parentes", quando comparado ao grupo de basefamiliar (G2).

A Tabela 3 apresenta a comparação entre G1 e G2 para as seguintes variáveis: número e impacto dos eventos estressores, indicadores de ajustamento e indicador geral de mau ajustamento. Para as análises da rede de apoio foram levados em conta o Indicador Geral de Mau Ajustamento, assim como as variáveis de Número e Impacto de Eventos Estressores.
Houve diferença estatisticamente significativa entre os grupos, sendo que o de base-rua apresentou maior média de eventos estressores e valores de impacto e pior indicador geral de mau ajustamento, comparado ao grupo de base-familiar. Os valores do indicador de mau ajustamento variaram entre os participantes de -6.32 (melhor ajustamento) a 9.17 (pior ajustamento).

\section{Efeito Moderador da Rede de Apoio na Influência dos Eventos Estressores (Número e Impacto) Sobre o Mau Ajustamento}

A análise de moderação foi realizada de acordo com o procedimento sugerido por Baron e Kenny (1986), com o objetivo de verificar se as características da rede de apoio (número de contatos total e por campo e fator de proximidade) moderam o efeito do número e impacto dos eventos estressores sobre o mau ajustamento. Quando ambas as variáveis preditora e moderadora são categóricas, os autores sugerem a realização de uma ANOVA fatorial $2 \mathrm{x}$ 2 em que a moderação é indicada por um efeito de interação destas variáveis. Assim, tanto a variável 


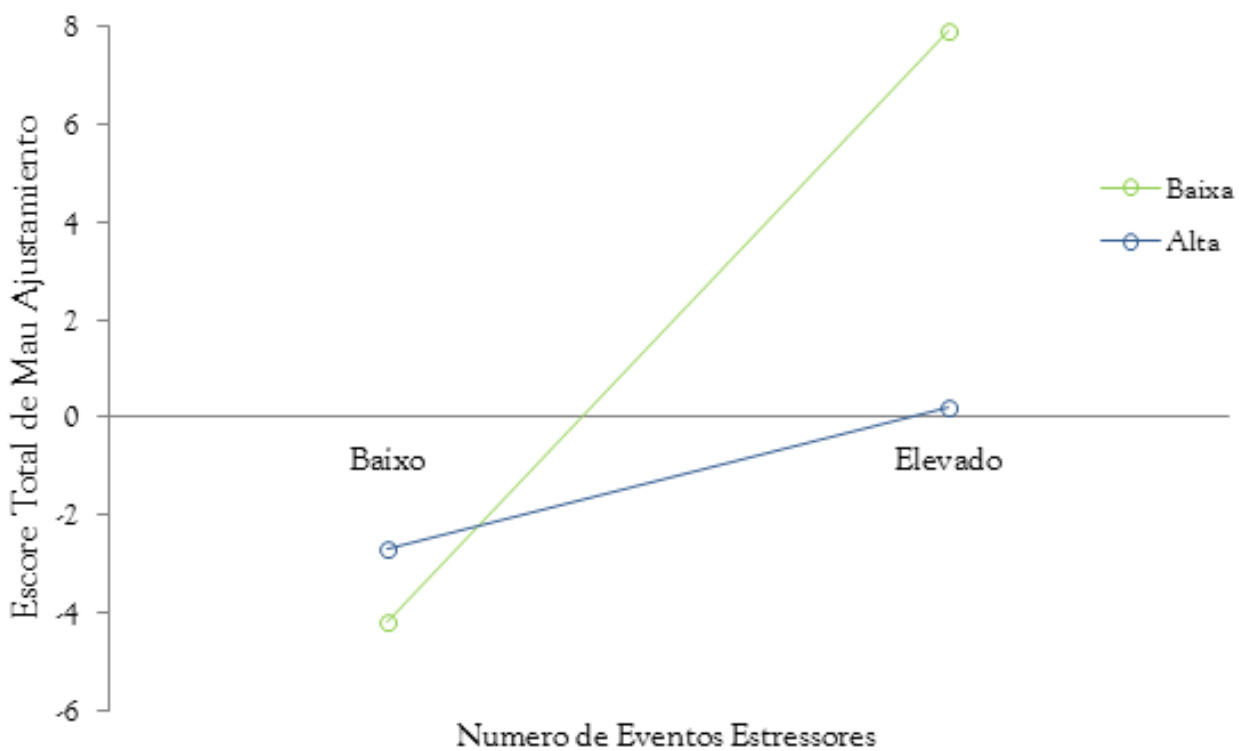

Figura 2. Proximidade familiar como moderadora da relação entre número de eventos estressores e o indicador geral de mau ajustamento

Fuente: elaboración propia.

preditora (número ou impacto de eventos estressores) quanto a moderadora (número total de contatos ou proximidade familiar) foram dicotomizadas a fim de representarem grupos de participantes com altos ( $25 \%$ da amostra com os valores mais elevados) e baixos valores ( $25 \%$ da amostra com os valores mais baixos) nas variáveis estudadas.

\section{Número de Eventos Estressores}

No total, foram realizadas dez análises de moderação (dada a existência de duas variáveis moderadoras e cinco campos a serem avaliados), sendo que apenas no fator de proximidade no campo família ocorreu uma interação com efeito significativo. Uma ANOVA fatorial do tipo 2 (alto vs. baixo nível de proximidade familiar) 2 (alto vs. baixo número de eventos estressores) em que o nível de mau ajustamento representou a variável dependente revelou efeitos principais significativos tanto para a proximidade familiar, $F(1.8)=5.3, p<0.05$, quanto para os eventos estressores, $F(1.8)=33.2, p<0.001$. Foi encontrado um efeito de interação significativo entre estas duas variáveis, $F(1.8)=11.7, p<0.001$. A descrição gráfica dessa interação mostrou que houve moderação da proximidade familiar no nível de ajustamento de indivíduos que vivenciam alto nível de eventos estressores. Ou seja, na presença de um alto nível de proximidade familiar, o impacto destes eventos sobre o mau ajustamento foi menor do que quando o nível de proximidade familiar era baixo (ver Figura 2).

\section{Impacto dos Eventos Estressores}

Nenhuma interação significativa foi verificada entre número total de contatos e fator de proximidade (para todo o mapa e nos quatro subcampos) com a variável de "impacto" de eventos estressores. Esse fato sugere que nenhuma característica do mapa dos cinco campos (seja com relação a todo o mapa ou por campos específicos) age moderando o impacto dos eventos estressores sobre o nível de mau ajustamento dos participantes.

\section{Discussão}

Com relação à rede de apoio dos participantes (ver Tabela 2), verificou-se que o maior número de contatos do grupo de base-rua foi mencionado no 
campo de instituição. Como parte desse campo, os adolescentes citaram nomes de amigos, educadores, monitores e coordenadores que fazem parte das instituições (albergue noturno e abrigo diurno). Já o maior fator de proximidade esteve relacionado ao campo de amigos, vizinhos e parentes. Os dados corroboram a ideia da existência de uma rede de apoio formada no contexto da rua em detrimento do contexto familiar e da escola, conforme descrito também por Bates e Toro (1999). A rede de apoio desse grupo parece estar restrita às instituições da rede de assistência. No grupo de base-familiar o maior número de contatos localizou-se no campo de amigos, vizinhos e parentes, enquanto o maior fator de proximidade foi verificado no campo de família. Quando comparados com relação ao fator de proximidade, apenas três diferenças emergiram: G1 apresentou maior escore total e para o campo de "amigos/vizinhos e parentes", comprovando o lugar de destaque dado por esse grupo aos amigos. A diferença na rede de apoio entre G1 e G2, portanto, mais que quantitativa (número de contatos), é qualitativa (fator de proximidade). Tal diferença é constatada durante a inserção ecológica com os dois grupos participantes. $\mathrm{O}$ grupo de base-rua faz mais referência ao grupo de amigos na busca de apoio afetivo e material e apresenta muitos relatos de violência/sofrimentos por membros de sua família. Os adolescentes do grupo de base-familiar, por sua vez, apresentam em geral uma visão mais positiva da sua família, mesmo descrevendo um maior número de contatos no campo de amigos/ vizinhos, característica típica da adolescência ( $\mathrm{Pa}$ palia \& Olds, 2000).

Orupo de base-rua apresentou maior média de número e impacto de eventos estressores (ver Tabela 3), que o de base-familiar. Muitos eventos estressores têm antecedido a migração para a rua, conforme relatado por vários autores (Morais, Neiva-Silva \& Koller, 2010; Rizzini, 1995; Rizzini \& Butler, 2003). Esses problemas foram mencionados nas entrevistas e se relacionam à violência familiar, a problemas no contexto escolar (problemas com professores, reprovação, abandono da escola, etc.) e a dificuldades financeiras. No entanto, como des- tacado por outros estudos (Ribeiro, 2001, 2003), na rua, a violência continua.

Neste ponto reside a "contradição" destacada por Noto et al. (2004) acerca da rua na vida dos adolescentes, bem como o lugar de destaque que a violência parece ter nas relações estabelecidas nos diferentes espaços que frequentam. Se por um lado, eles informam buscar a rua como uma alternativa para se libertarem dos constantes castigos, ameaças físicas e agressões verbais vivenciados na sua casa, na rua, continuam a sofrer estes e/ou outros tipos de violência (ameaça com armas; agressão física; ameaças de abuso sexual; estupro; etc.). Na rua, os agressores não eram mais os próprios familiares, mas os companheiros, integrantes de gangs, policiais, e a população em geral que os exclui e tem medo deles. Acerca da violência vivenciada pelos adolescentes em situação de rua, Ribeiro e Ciampone (2001) afirmaram que "durante as suas trajetórias de vida eles viveram em muitas situações diferentes, mas em todas a violência parece ter sido a protagonista" (p. 47).

Os resultados dos grupos nos indicadores de ajustamento mostraram que as médias de sintomas físicos, uso de drogas, comportamento suicida, comportamento sexual de risco, afeto positivo e afeto negativo foram sempre maiores no grupo de base-rua, quando comparado ao de base-familiar. Consequentemente, o indicador geral de mau ajustamento também foi maior para o grupo de base-rua (ver Tabela 3). O contexto de adversidade vivenciado especialmente pelo grupo de base-rua (G1), revela-se na realidade de vida na rua, incluindo uso de drogas, sexo de sobrevivência, perda de apoio, abrigo seguro e necessidades básicas de higiene e colocam essa população em situação de risco para muitas consequências negativas de saúde. Dentre as quais se citam: dependência química, DSTs, infecção pelo HIV, má nutrição, déficits de crescimento, lesões por acidentes, gravidez indesejada e morte prematura resultante de suicídio e homicídio (Morais \& Koller, 2010; Morais, Koller \& Raffaelli, 2010).

O fator de proximidade na família agiu como um importante moderador do efeito do número de eventos estressores sobre o mau ajustamento (ver Figura 2). Isso significa que, na presença de muitos 
eventos estressores, quanto maior for o fator de proximidade familiar, menor o mau ajustamento. De acordo com Fergus e Zimmerman (2005) e Luthar, Cicchetti, e Becker (2000), esse modelo de ação é conhecido como "protetivo-reativo", já que na presença da rede de apoio familiar o resultado adverso (mau ajustamento) ainda se mostra presente, mas em menor intensidade. Esse resultado está de acordo com a literatura, a qual destaca que a rede de apoio, especialmente aquela formada pela família, é um fator fundamental para o processo de adaptação a situações de estresse e de suscetibilidade a distúrbios físicos e emocionais (Masten \& Garmezy, 1985; Rutter, 1985-1987; Samuelsson, Thernlund \& Ringström, 1996).

\section{Considerações Finais}

Os resultados do presente estudo corroboram empiricamente a importância da "proximidade" familiar como um fator de proteção na vida de adolescentes que vivem em situação de vulnerabilidade social. Mais especificamente, sobre a vida daqueles/as que estão submetidos a um maior número de eventos estressores. Nesse sentido, esse artigo pretende ser uma contribuição teórica importante, pois testa estatisticamente uma hipótese teórica.

Nenhum estudo que testava a associação entre rede de apoio e mau ajustamento, a partir do Mapa dos Cinco Campos, foi identificado até então. Quase sempre, as pesquisas que utilizam esse instrumento o utilizam para descrever as características da rede de apoio dos participantes (Hoppe, 1998; Siqueira, Betts \& Dell'Aglio, 2006; Siqueira et al., 2009). A utilização deste instrumento com adolescentes em situação de vulnerabilidade social (na rua ou na família) mostrou-se apropriado, pela riqueza de dados produzidos e pelo aspecto lúdico e terapêutico que sua aplicação possibilita. Durante a aplicação do Mapa, foi frequente a expressão de sentimentos e relatos de vida, que talvez demorassem mais tempo para serem expressos, caso apenas a entrevista tivesse sido utilizada.

À luz desses resultados, confirma-se a necessidade de programas e políticas sociais que tenham a família como foco de atenção e interesse (ver Morais, Paludo, \& Koller, 2010). De acordo com Serapioni (2005), a retomada da família como referência das políticas públicas é justificada como a estratégia mais adequada (ao lado das intervenções sociais tradicionais - saúde, educação, habitação, renda, etc.) para o desenvolvimento de programas sociais efetivos de enfrentamento das diversas situações que implicam em vulnerabilidade social (tais como, pobreza, violência, situação de rua etc.). Além disso, o foco na família está relacionado com a visão de que programas e políticas públicas com foco no indivíduo tendem ao fracasso, assim como as intervenções pontuais, compensatórias e assistencialistas (Carvalho \& Almeida, 2003; Serapioni, 2005).

Embora no discurso oficial, as iniciativas de proteção à família recebam um apoio cada vez maior, verifica-se que as mesmas ainda possuem muitas limitações. Estas se referem ao fato de que atingem apenas uma reduzida parcela de seu público-alvo, deixando, portanto de fora um amplo contingente de jovens e famílias empobrecidos e vulnerabilizados, os quais mesmo não sendo considerados como em uma situação extrema de pobreza, encontram-se excluídos do acesso aos suportes sociais. A escassez de acesso a uma rede de suporte social, por sua vez, faz com que a família perca gradativamente sua capacidade de funcionar como amortecedor da crise e como mecanismo de proteção de seus componentes, o que leva não apenas à deterioração das suas condições de vida, como também afeta e ameaça a própria unidade e coesão familiar (Carvalho \& Almeida, 2003).

Tão importante quanto o suprimento de condições materiais e econômicas às famílias é a existência de programas sociais que visem a um acompanhamento mais sistemático dessas famílias. As propostas assistencialistas vigentes até então apenas reforçaram a percepção dessas famílias como incapazes e desqualificadas para o cuidado de seus filhos. Urge uma nova concepção de família, a qual esteja baseada não apenas nas suas vulnerabilidades e fragilidades, mas que as reconheça também em suas próprias potencialidades e recursos e que atue fortalecendo-os (Gomes $\&$ Pereira, 2005). 
Por fim, merecem destaque algumas limitações do presente estudo. A primeira delas diz respeito ao pequeno número de participantes do grupo de base-rua, sobretudo de meninas. Apesar do extenso período de tempo passado pela equipe de pesquisa nas instituições destinadas a essa população, verifica-se que houve um esvaziamento das mesmas durante o período em que a pesquisa ocorreu. Esse fato, no entender dos técnicos e dirigentes das instituições está relacionado ao fato de que muitos meninos e meninas "potenciais" para a situação de rua e frequência das ruas no centro da cidade estariam ficando em suas comunidades de origem, quase sempre se envolvendo com o tráfico. De fato, essa hipótese faz muito sentido, sobretudo depois que foram capturados - não um apenas, mas vários - relatos de adolescentes que disseram estar no "centro", porque estavam fugindo de traficantes e/ ou de inimizades nas suas comunidades de origem. Outra limitação diz respeito à perda de respostas dos participantes da rua nos instrumentos que avaliavam o ajustamento. Uma vez que esses instrumentos estavam contidos na entrevista que era a última parte a ser feita no processo de coleta de dados, e dada à alta dinamicidade da vida desses adolescentes (um dia estão na instituição, outro dia não estão mais), muitos participantes acabaram não respondendo a esses instrumentos que ficavam para o final. No grupo com base na família, uma vez que a sua frequência à instituição onde a coleta foi realizada era mais contínua, essa perda foi menor.

Em termos de estudos futuros, sugere-se que análises de moderação sejam realizadas para cada subgrupo e não apenas considerando a amostra total. Dessa forma, se obterá uma análise mais detalhada de como a rede de apoio age moderando o efeito do número/impacto dos eventos estressores sobre o mau ajustamento em cada grupo. Essa informação é muita necessária, a fim de se estabelecer semelhanças e diferenças na dinâmica desenvolvimental de cada grupo. Outra importante sugestão é a de que diferentes fatores de proteção, relacionados à dimensão mais pessoal/individual - os chamados assets (por exemplo: habilidades sociais, auto-eficácia e auto-estima) tenham o seu papel moderador testado.

\section{Referências}

Baron, R. M. \& Kenny, D. A. (1986). The moderatormediator variable distinction in social psychological research: Conceptual, strategic, and statistical considerations. Journal of Personality and Social Psychology, 51, 1173-1182.

Bates, D. S. \& Toro, P. A. (1999). Developing measures to assess social support among homeless and poor people. Journal of Community Psychology, 27, 137-156.

Brito, R. \& Koller, S. H. (1999). Desenvolvimento humano e redes de apoio social e afetivo. In A. M. Carvalho (Ed.). O mundo social da criança: $\mathrm{Na}$ tureza e cultura em ação (pp. 115-129). São Paulo: Casa do Psicólogo.

Bronfenbrenner, U. (1989). Ecological systems theory. In R. Vasta (Ed.), Annals of Child Development, 6, 187-249.

Bronfenbrenner, U. (1996). A ecologia do desenvolvimento humano: Experimentos naturais e planejados. Porto Alegre: Artes Médicas (originalmente publicado em 1979).

Bronfenbrenner, U. (2005). Making human beings humans. Thousand Oaks: SAGE.

Bronfenbrenner, U. \& Morris, P. (1998). The ecology of developmental processes. In W. Damon (Ed.), Handbook of child psychology (Vol.1, pp. 993-1027). New York, NY: John Wiley \& Sons.

Carvalho, I. M. M. \& Almeida, P. H. (2003). Família e proteção social. São Paulo em Perspectiva, 17, 109-122.

Cecconello, A. M. \& Koller, S. H. (2003). Inserção ecológica na comunidade: Uma proposta metodológica para o estudo de famílias em situação de risco. Psicologia Reflexão e Crítica, 16, 515-524.

Cohen, S. \& Wills, T. A. (1985). Stress, social support, and the buffering hypothesis. Psychological Bulletin, 98, 310-357.

Cowan, P. A., Cowan, C. P. \& Schulz, M. S. (1996). Thinking about risk and resilience in families. In E. M. Hetherington \& E. A. Blechman (Eds.), Stress, coping and resiliency in children and families (pp. 1-38). New Jersey: Erlbaum.

Eschiletti-Prati, L. E., Couto, M. C. P. P., Moura, A., Poletto, M. \& Koller, S. (2008). Revisando a in- 
serção ecológica: uma proposta de sistematização. Psicologia Reflexão e Crítica, 21,160-169.

Farrel, A. D. \& White, K. S. (1998). Peer influences and drug use among urban adolescents: family structure and parent adolescent relationship as protective factors. Journal of Consulting and Clinical Psychology, 66, 248-258.

Fergus, S. \& Zimmerman, M. A. (2005). Adolescent resilience: a framework for understanding healthy development in the face of risk. Annual Reviews of Public Health, 26, 39-419.

Giacomoni, C. (2002). Bem-estar subjetivo infantil: Conceito de felicidade e construção de instrumentos para avaliação. Tese de Doutorado Inédita, Curso de Pós-Graduação em Psicologia do Desenvolvimento. Universidade Federal do Rio Grande do Sul, Porto Alegre, RS.

Gomes, M. A. \& Pereira, M. L. D. (2005). Família em situação de vulnerabilidade social: Uma questão de políticas públicas. Ciência e Saúde Coletiva, 10, 257-363.

Hoppe, M. (1998). Redes de apoio social e afetivo de crianças em situação de risco. Dissertação de Mestrado não-publicada, Curso de Psicologia do Desenvolvimento, Universidade Federal do Rio Grande do Sul.

Jessor, R., Van Den Bos, J., Vanderryn, J., Costa, F. M. \& Turbin, M. S. (1995). Protective factors in adolescent problem behavior: Moderator effects and developmental Change. Developmental Psychology, 31, 923-933.

Johnson, K. D., Whitbeck, L. B. \& Hoyt, D. R. (2005). Predictors of social network composition among homeless and runaway adolescents. Journal of Adolescence, 28, 231-248.

Kristensen, C. H., Dell'Aglio, D. D., Leon, J. S. \& D'Incao, D. B. (2004). Análise da freqüência e do impacto de eventos estressores em uma amostra de adolescentes. Interação, 8, 45-55.

Laurent, J., Catanzaro, S. J., Joiner, T. E., Rudolph, K. D., Potter, K. I., Lambert, S., Osborne, L. \& Gathright, T. (1999). A measure of positive and negative affect for children: Scale development and preliminary validation. Psychological Assessment, 11, 326-338.

Luthar, S. S., Cicchetti, D. \& Becker, B. (2000). The construct of resilience: a critical evaluation and guidelines for future work. Child Development, 71, 543-562.

Martins, P. C. M. (2004). Proteção de crianças e jovens em itinerários de risco: Representações sociais, modos e espaços. Tese de Doutorado não publicada, Instituto de Estudos da Criança, Universidade do Minho, Portugal.

Masten, A. S. \& Garmezy, N. (1985). Risk, vulnerability and protective factors in developmental psychopathology. In B. B. Lahey \& A. E. Kazdin (Eds.), Advances in clinical child psychology (Vol. 8, pp.152). New York: Plenum Press.

Morais, N. A. \& Koller, S. H. (2010). A saúde de crianças e adolescentes em situação de rua (pp. 235-262). In N. A. de Morais, L. Neiva-Silva \& S. H. Koller (Eds.), Endereço Desconhecido: Crianças e adolescentes em situação de rua. São Paulo: Casa do Psicólogo.

Morais, N. A., Koller, S. H. \& Raffaelli, M. (2010). Eventos estressores e indicadores de ajustamento entre adolescentes em situação de vulnerabilidade social no Brasil. Universitas Psychologica, 9, 787-806.

Morais, N. A., Neiva-Silva, L. \& Koller, S. H. (2010). Endereço desconhecido: crianças e adolescentes em situação de rua. São Paulo: Casa do Psicólogo.

Morais, N. A., Paludo, S. \& Koller, S. H. (2010). A família de crianças e adolescentes em situação de rua. In N. A. de Morais, L. Neiva-Silva \& S. H. Koller (Eds.), Endereço desconhecido: crianças e adolescentes em situação de rua (pp. 177-197). São Paulo: Casa do Psicólogo.

Noto, A. R., Galduróz, J. C. F., Nappo, S. A., Carlini, C. M. A., Moura, Y. G. \& Carlini, E. A. (2004). Levantamento nacional sobre o uso de drogas entre crianças e adolescentes em situação de rua nas 27 capitais brasileiras (2003). São Paulo: UNIFESP/ CEBRID.

Papalia, D. \& Olds, S. (2000). Desenvolvimento Humano. Porto Alegre: Artmed.

Ribeiro, M. O. (2001). A criança de rua tem família: Uma família em crise. Revista Brasileira de Crescimento e Desenvolvimento Humano, 11(1), 35-47.

Ribeiro, M. O. (2003). A rua: Um acolhimento falaz às crianças que nela vivem. Revista Latino-americana de Enfermagem, 11, 622-629. 
Ribeiro, M. O. \& Ciampone, M. H. T. (2001). Homeless children: The lives of a group of Brazilian street children. Journal of Advanced Nursing, 35(1), 42-49.

Rizzini, I. (1995). Deserdados da sociedade: Os "meninos de rua" da América Latina. Rio de Janeiro: CESPI/ USU.

Rizzini, I. \& Butler, U. M. (2003). Crianças e adolescentes que vivem e trabalham nas ruas: revisitando a literatura. In I. Rizzini (Ed.), Vida nas ruas: Crianças e adolescentes nas ruas: trajetórias inevitáveis? (pp. 17-44). Rio de Janeiro/São Paulo: PUC-Rio/Loyola.

Rutter, M. (1987). Psychosocial resilience and protective mechanisms. American Journal of Orthopsychiatry, 57(3), 316-331.

Rutter, M. (1985). Resilience in the face of adversity: Protective factors and resistance to psychiatric disorder. British Journal of Psychiatry, 147, 598-611.
Samuelson, M., Thernlund, G. \& Ringström, J. (1996). Using the five field map to describe the social network of children: A methodological study. International Journal of Behavioral Development, $19,327-345$.

Serapioni, M. (2005). O papel da família e das redes primárias na reestruturação das políticas sociais. Ciência e Saúde Coletiva, 10, 243-253.

Siqueira, A. C., Betts, M. K. \& Dell'Aglio, D. D. (2006). Redes de apoio social e afetivo de adolescentes institucionalizados. Interamerican Journal Psychology, 40(2), 149-158.

Siqueira, A. C., Tubino, C. L., Schwarz, C. \& Dell' Aglio, D. D. (2009). Família e institucionalização: Percepção das figuras parentais na rede de apoio de crianças e adolescentes institucionalizados. Arquivos Brasileiros de Psicologia, 61, 176-190. 
